\title{
Nichtlineare Analyse von LC-TANK VCOs unter Berücksichtigung parasitärer Substrateffekte
}

\author{
J. Przytarski, J.-K. Bremer, and W. Mathis \\ Leibniz Universität Hannover, Institut für Theoretische Elektrotechnik, Appelstr. 9A, 30167 Hannover, Germany
}

\begin{abstract}
Zusammenfassung. In dieser Arbeit wird ein Konzept vorgestellt, die Schaltungsarchitektur des LC-Tank-VCOs im höherdimensionalen Zustandsraum zu modellieren. Dabei wurde die VCO-Schaltung auf dominante nichtlineare und asymmetrische Effekte näher untersucht. Das entwickelte Modell bildet die Grundlage für nachfolgende parameterabhängige Analysen der Oszillatorschaltung mittels der Andronov-Hopf Bifurkationsanalyse. Zu diesem Zweck wurde ein höherdimensionales Modell entwickelt, welches für die Bifurkationsanalyse auf ein System 2. Ordnung reduziert werden muss. Als mathematisches Hilfsmittel zur Ordnungsreduktion des Systems wird das Verfahren der Zentrumsmannigfaltigkeit verwendet. Das Differentialgleichungssystem wird sehr schaltungsnah aufgestellt, daraus resultiert der Vorteil einer genaueren Repräsentation des physikalischen Verhaltens der Schaltung. Ziel ist es möglichst viele Effekte die sich auf das Verhalten des VCOs auswirken können, mit zu berücksichtigen.
\end{abstract}

\section{Einleitung}

Generell werden die Anforderungen an moderne VCOs und die damit in Relation stehenden Spezifikationen immer höher, besonders die Phasenrauschspezifikationen sind von großer Bedeutung. Bei dem Design von Oszillatorschaltungen bezieht man sich oft auf Erfahrungswerte und gelangt häufig nur durch viele Simulationen zu einem guten Ergebnis. Grund dafür ist die Komplexität und das nichtlineare Verhalten der Oszillatorschaltung. $\mathrm{Zu}$ den wichtigsten Spezifikationen gehören hierbei die Frequenz, die Amplitude und das Phasenrauschen. Folglich wäre es wünschenswert, wenn diese Kriterien schon vor der Schaltungssimulation in einem gewissen Bereich vorhersagbar wären. Erschwerend kommen im Zuge der steigenden Integration parasitäre Effekte durch das Substrat hinzu. Zu diesem Zweck ist eine parameterabhängige Untersuchung an einem Modell, welches das

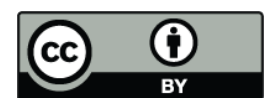

Correspondence to: J.-K. Bremer (jbre@tet.uni-hannover.de)
Schaltungsverhalten möglichst physikalisch genau repräsentiert, nützlich. In diesem Zusammenhang sind in den vergangen Jahren verschiedene Arbeiten zur parameterabhängigen Untersuchung von Oszillatorschaltungen und deren Entwurf durchgeführt worden (vgl. Keidies, 1995; Prochaska, 2005 und Bremer, 2009). In diesen Arbeiten wird bei den Analysen der Schaltungen der in (Buonomo, 2004) vorgestellten 2-dimensionale Modellierungsansatz verwendet. Grundgedanke dieses Ansatzes ist den LC-Tank bestehend aus vier Energiespeichern, durch ausnutzen von Symmetriebedingungen, auf ein 2-dimensionales nichtlineares Differentialgleichungsystem zu reduzieren. Der Vorteil des 2-dimensionalen Modellierungsansatzes ist die relativ einfache mathematische Handhabbarkeit. Allerdings berücksichtigt dieser Ansatz keine asymmetrischen und nur eingeschränkt die nichtlinearen Effekte, wie die Einkopplungen der Spannungsschwankungen am Fußpunkt des kreuzgekoppelten Transistorpaares in den Tank, den asymmetrischen Stromfluß im Tank durch gegenseitige Potentialverläufe der Varaktoren oder zusätzliche Strompfade durch strukturelle Erweiterungen. Da eine Literaturrecherche zu diesem Zeitpunkt für den LC-Tank keinen alternativen höhrdimensionalen Modellierungsansansatz ergab, wird in dieser Arbeit ein Konzept vorgestellt, um die zuvor erwähnten asymmetrischen Effekte mit zu berücksichtigen.

\section{2-dimensionale VCO-Modellierung}

In Abb. 1 ist das Schaltbild einer gängigen NMOS-LC-Tank VCO-Schaltung und das daraus abgeleitete 2-dimensionale Ersatzschaltbild dargestellt (vgl. Buonomo, 2004). Die Aufteilung der Ströme in der Schaltung wird als rein symmetrisch angenommen, dadurch können die Energiespeicher, die Transistoren und ihre Verluste zusammengefasst werden, wie es in der Abb. 1 gezeigt ist.

Gesucht ist für die vollständige Zusammenfassung letztendlich noch der Zusammenhang zwischen dem differentiellen Strom durch das kreuzgekoppelte Transistorpaar $i_{d}$ in Abhängigkeit von Oszillatoramplitude $v_{t}$. Dieser Zusammenhang repräsentiert im Modell das Verhalten des kreuzgekop-

Published by Copernicus Publications on behalf of the URSI Landesausschuss in der Bundesrepublik Deutschland e.V. 

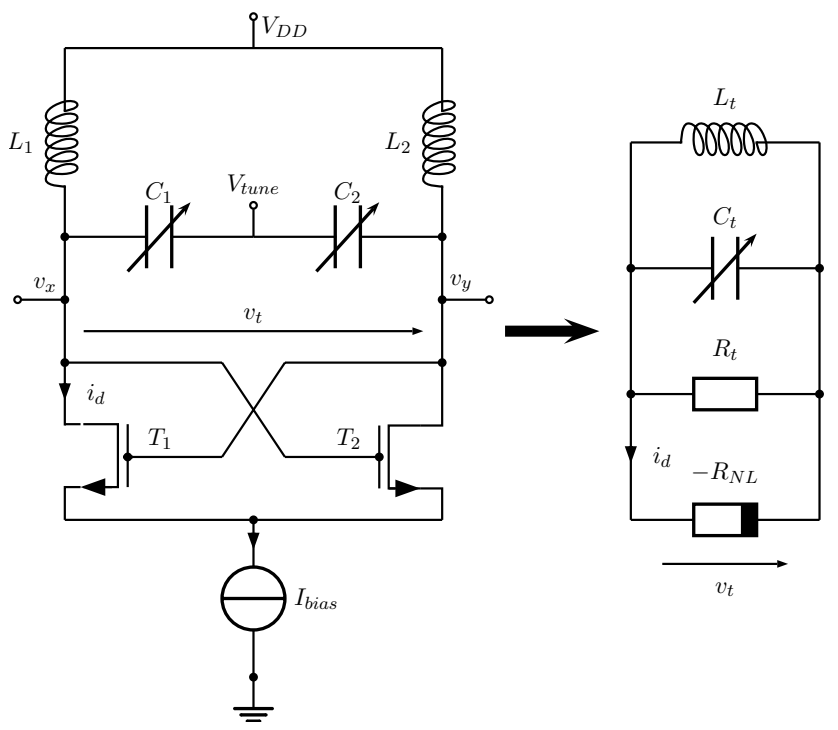

Abb. 1. Ersatzschaltbild für die LC-Tank VCO-Architektur.

pelten Transitorpaares. Hier wird zunächst die Annahme getroffen, dass sich beide Transistoren in Sättigung befinden, also gilt für den MOSFET-Drainstrom $i_{D}=K\left(V_{G S}-V_{T}\right)^{2}$. Damit ergibt sich mit $V_{n}=\sqrt{2 I_{\text {bias }} / K}$ der Zusammenhang

$$
i_{d}\left(v_{t}\right)=-I_{\text {bias }} \frac{v_{t}}{V_{n}} \sqrt{1-\frac{v_{t}^{2}}{V_{n}^{2}}},
$$

was einem negativen Widerstand entspricht (vgl. Buonomo, 2004). Für das Ersatzschaltbild lässt sich nun leicht das folgende Differentialgleichungssystem

$$
\left(\begin{array}{c}
\frac{d v_{t}}{d t} \\
\frac{d i_{L}}{d t}
\end{array}\right)=\left(\begin{array}{cc}
-\frac{1}{R_{t} C_{t}} & -\frac{1}{C_{t}} \\
\frac{1}{L_{t}} & 0
\end{array}\right)\left(\begin{array}{l}
v_{t} \\
i_{L}
\end{array}\right)-\left(\begin{array}{c}
\frac{i_{d}\left(v_{t}\right)}{C_{t}} \\
0
\end{array}\right)
$$

vollständig aufstellen (vgl. Prochaska, 2005).

\section{Asymmetrische und nichtlineare Effekte}

Aufgrund der Annahme idealer Symmetrie in der Stromverteilung der Schaltung ist die Berücksichtigung asymmetrischer Effekte nicht gegeben. In diesem Abschnitt soll ein kurzer Überblick über asymmetrische und nichtlineare Effekte in der LC-Tank Schaltung gegeben werden. Im Anschluß wird dann ein alternatives Modellierungskonzept, das diese Effekte mitberücksichtigen kann, vorgestellt.

\subsection{Substrateffekte}

Das Substratmaterial besitzt u.a. parasitäre resistive und kapazitive Effekte. Bei der integrierten Realisierung eines LCTank VCOs können die einzelnen Bauelemente also nicht als ideal angenommen werden. Die Spule gehört zu den sensibelsten Elementen des VCOs und besitzt dazu eine große

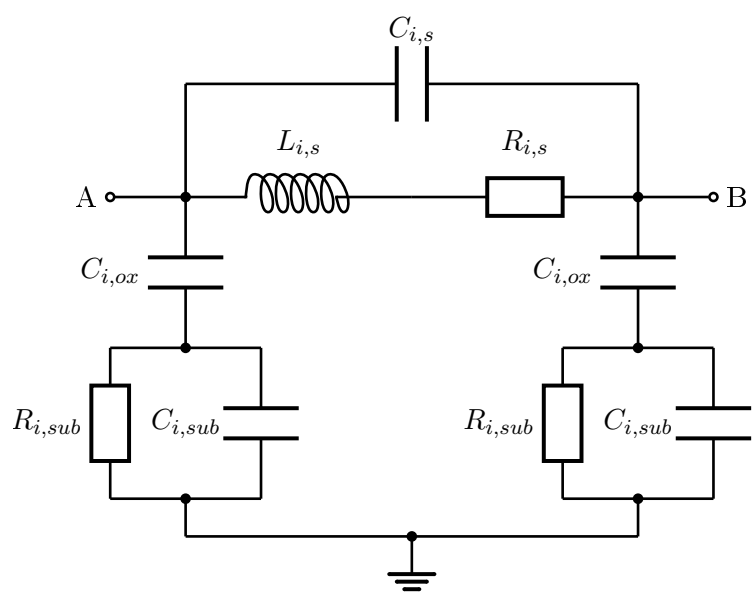

Abb. 2. Modell einer integrierten Spule.

Kontaktfläche zum Substrat. Eine Zusammenfassung der parasitären Effekte ist exemplarisch für die Spule in dem in Abb. 2 dargestellten Modell gezeigt (vgl. Yue, 2000).

Die Oxidkapazität zwischen Spule und Substrat ist mit $C_{o x}$ bezeichnet, das Substrat selbst wird durch $C_{\text {sub }}$ und $R_{\text {sub }}$ modelliert. Durch diese parasitären Elemente kann es zu Störeinkopplungen von anderen Schaltungsblöcken, insbesondere bei Mixed-Signal Schaltungen, kommen. Die Degradierung der Phasenrauscheigenschaften aufgrund Störungseinkopplungen durch das Substrat werden von Mendez (2005) experimentell ermittelt und diskutiert. Weiterhin werden in Soens (2005) Simulationsmethodiken vorgestellt, um die Auswirkungen des Substrats auf die VCOPhasenrauscheigenschaften in einem gewissen Rahmen vorhersagen zu können. Um gezielte Aussagen auf Schaltungsebene treffen zu können, wäre es hilfreich wenn diese parasitären Elemente als direkt zugängliche Größen im Differentialgleichungssystem vorhanden sind. Deshalb wird in Abschnitt 4 ein höherdimensionaler Modellierungsansatz vorgestellt.

\subsection{Kreuzgekoppeltes Transistorpaar}

Bei der in Abschnitt 2 gezeigten 2-dimensionalen Modellierung ist das Ersatzschaltbild stark von der ursprünglichen Struktur der Schaltung entkoppelt. Dies führt zwar zu einer erheblichen Vereinfachung der Differentialgleichungssystems, das Ersatzschaltbild kann aber leider das kreuzgekoppelte Transistorpaar nicht vollständig erfassen. Das kreuzgekoppelte Transistorpaar ist im Modell lediglich ein negativer Widerstand, der unabhängig ist von der Spannung über der Bias-Quelle und der einen symmetrischen Stromfluß besitzt. Allerdings überträgt das kreuzgekoppelte Transistorpaar die Spannungsschwankungen am Fusspunkt des Transistorpaares in den Tank und der Stromfluß durch das Transistorpaar ist nicht rein symmetrisch. In Abb. 3 ist in der untersten Kurve diese Fusspunktspannung im Verlauf über der Zeit 


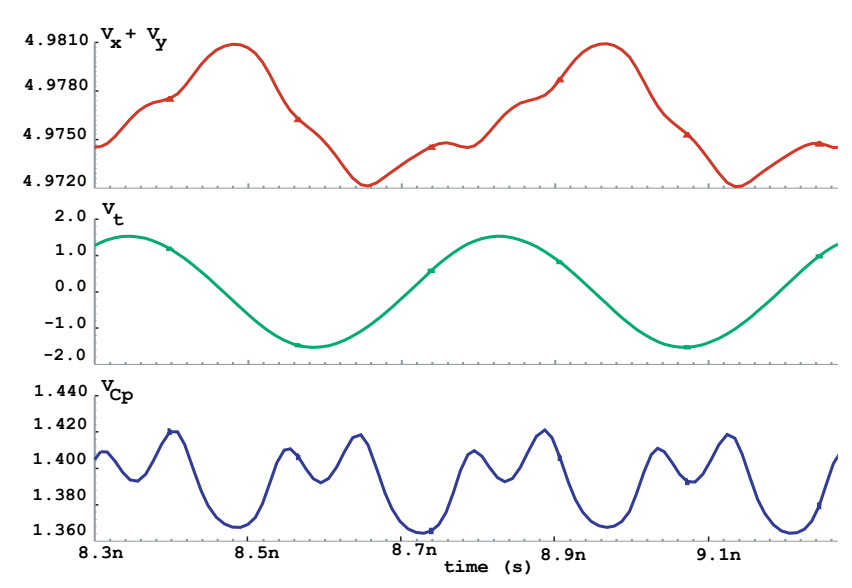

Abb. 3. Transiente Darstellung der Spannungen $v_{x}+v_{y}, v_{t}$ und $v_{C p}$.

dargestellt. Die oberste Kurve zeigt die Addition der Knotenspannungen $v_{x}+v_{y}$. Wäre die Arbeitsweise des kreuzgekoppelten Transistorpaares rein differentiell und symmetrisch müsste diese Summe konstant $2 \cdot V_{D D}$ sein. Die Oszillatoramplitude ist in der mittleren Kurve zu erkennen.

Auswirkungen hat dieses Verhalten auf die Verzerrungseigenschaften des Oszillatorausgangssignals durch AM-FMKonversion aufgrund der nichtlinearen Varaktorkennlinie (vgl. Hegazi, 2003), denn der Arbeitspunkt der Varaktoren ist nicht konstant.

\subsection{Strukturelle Erweiterungen}

In der Arbeit von Hajimiri (1999) wurde als strukturelle Erweiterung der LC-Tank VCO Schaltung eine Filterkapazität parallel zur Bias-Quelle vorgestellt (vgl. Abb. 4). Diese Filterkapazität soll die Spannung über der Biasquelle glätten und somit die Phasenrauscheigenschaften der Schaltung verbessern. Das Einbeziehen dieser Erweiterung in das aus Abschnitt 2 bekannte 2-dimensionale Modell ist allerdings nicht ohne weiteres möglich, wodurch die parameterabhängige Analyse so nicht anwendbar ist. Da die zusätzliche Filterkapazität u.a. Einfluß auf Frequenz und Phasenrauschen des LC-Tanks hat, ist die Einbeziehung in ein Modell für die Optimierung der Designparameter bezüglich der Spezifikation sinnvoll. Aus diesem Grunde wird im nächsten Abschnitt ein alternativer Modellierungsansatz vorgestellt.

\section{Höherdimensionaler Modellierungsansatz}

Um eine schaltungsnahe Modellierung des VCOs zu erhalten, werden nun die Netzwerkgleichungen der Schaltung direkt aufgestellt. Damit ist auch die Erweiterbarkeit sofort gegeben. Ziel ist es möglichst viele Effekte die sich auf das

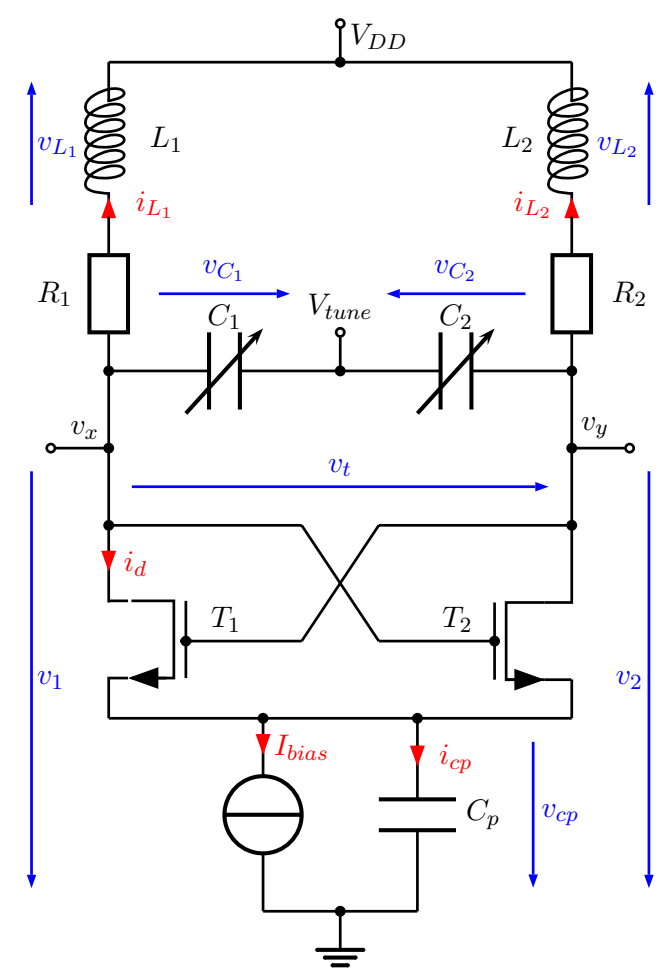

Abb. 4. VCO-Schaltung mit Filterkapazität.

Verhalten des VCOs auswirken können, mit zu berücksichtigen. In Abb. 4 ist das Schaltbild eines NMOS LC-TankOszillators mit Filterkapazität und die für das Aufstellen des zugehörigen Differentialgleichungssystems eingeführten Strom- und Spannungsbeziehungen gezeigt.

Das Differentialgleichungssystem ergibt sich somit zu

$$
\begin{aligned}
\frac{d v_{C_{p}}}{d t}= & \frac{1}{C_{p}} \cdot\left[K \cdot\left(v_{2}-v_{C_{p}}-V_{T H}\right)^{2}\right. \\
& \left.+K \cdot\left(v_{1}-v_{C_{p}}-V_{T H}\right)^{2}-I_{\text {bias }}\right] \\
\frac{d i_{L_{1}}}{d t}= & \frac{v_{1}-V_{D D}-i_{L_{1}} \cdot R_{1}}{L_{1}} \\
\frac{d i_{L_{2}}}{d t}= & \frac{v_{2}-V_{D D}-i_{L_{2}} \cdot R_{2}}{L_{2}} \\
\frac{d v_{1}}{d t}= & \frac{1}{C_{1}} \cdot\left[-K \cdot\left(v_{2}-v_{C_{p}}-V_{T H}\right)^{2}-i_{L_{1}}\right] \\
\frac{d v_{2}}{d t}= & \frac{1}{C_{2}} \cdot\left[-K \cdot\left(v_{1}-v_{C_{p}}-V_{T H}\right)^{2}-i_{L_{2}}\right] .
\end{aligned}
$$

Die Verluste der Schaltung sind in diesem Modellansatz exemplarisch in den Serienwiderständen der Spulen, $R_{1}$ und $R_{2}$, zusammengefasst. Um die Komplexität der Analyse zu reduzieren, wurde für die Varaktoren zunächst eine konstante Kapazität angenommen. Das System wird unter Anwendung von Verfahren aus der Theorie nichtlinearer dynamischer Systeme nun weiter untersucht. 


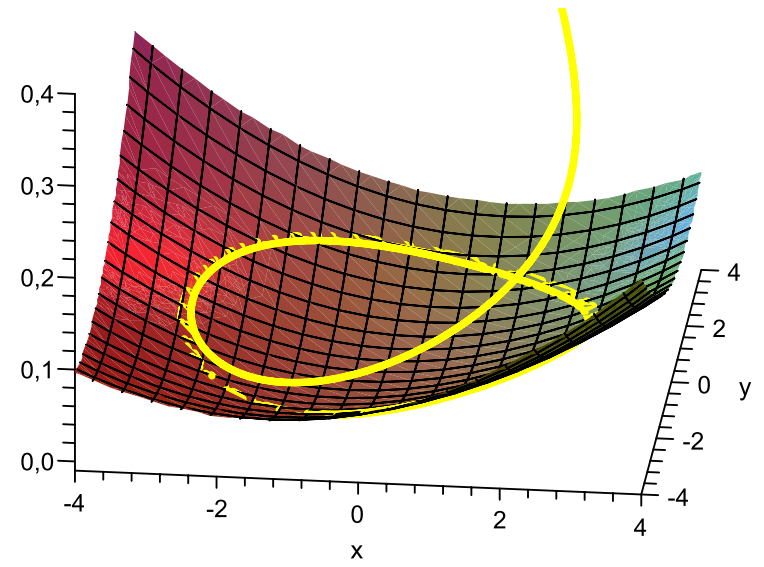

Abb. 5. Einlaufen der Lösung in einen Grenzzyklus im 3-dimensionalen Zustandsraum.

\section{Ordnungsreduktion}

Das zuvor aufgestellte Modell soll nun für den parameterabhängigen Oszillatorentwurf verwendet werden. Zunächst wird zu diesem Zweck für die spätere Andronov-HopfBifurkationsanalyse der Bifurktionsparameter $\mu$ eingeführt. Damit ergibt sich das parameterabhängige Differentialgleichungssystem in allgemeiner Form zu

$$
\dot{\mathbf{x}}=\mathbf{A}(\mu) \cdot \mathbf{x}+\tilde{f}(\mathbf{x}, \mu) .
$$

Es handelt sich bei dem System um eine Oszillatorschaltung, daher treten bei stabiler Oszillation nicht-hyperbolische Eigenwerte und Grenzzyklen auf. Ein Grenzzyklus ist eine geschlossene Trajektorie im Raum und kann deshalb immer in eine Fläche eingebettet werden (vgl. Abb. 5). Für die Approximation dieser Fläche wird hier das Verfahren der Zentrumsmannigfaltigkeit angesetzt (vgl. Guckenheimer, 1983). Dadurch kann der Grenzzyklus auf eine Ebene projiziert, folglich das System auf 2. Ordnung reduziert, werden.

\subsection{Zentrumsmannigfaltigkeit}

Die gesuchte Zentrumsmannigfaltigkeit ist die invariante Mannigfaltigkeit, die den linearen Eigenraum mit den Eigenwerten $\mathrm{Re}=0$ tangiert. Das bedeutet die Berechnung der Zentrumsmannigfaltigkeit findet im stationären Punkt, in dem ein Grenzzyklus existiert, statt. Der stationäre Lösungsvektor ergibt sich in Abhängigkeit des Parameters $K$ zu

$$
\mathbf{x}_{s}=\left(\begin{array}{c}
v_{c p_{s}} \\
i_{L 1_{s}} \\
i_{L 2_{s}} \\
v_{1_{s}} \\
v_{2_{s}}
\end{array}\right)=\left(\begin{array}{c}
k_{1} \cdot \frac{1}{\sqrt{K}} V \\
-I_{\text {bias }} \\
-I_{\text {bias }} \\
V_{D D}-\frac{1}{2} I_{\text {bias }} R_{1} \\
V_{D D}-\frac{1}{2} I_{\text {bias }} R_{2}
\end{array}\right),
$$

mit $k_{1}=-V_{T H}+V_{D D}-\frac{1}{2} I_{\text {bias }} R-\frac{1}{2} \sqrt{2 I_{\text {bias }}}$. Für die Bifurkationsanalyse muss das System in der Normalform

$$
\dot{\mathbf{x}}=\left(\begin{array}{cccc}
0 & -\omega & 0 & \cdots \\
\omega & 0 & 0 & \cdots \\
0 & 0 & \lambda_{s} & \cdots \\
\vdots & \vdots & \vdots & \ddots
\end{array}\right) \cdot \mathbf{x}_{s}+\tilde{f}(\mathbf{x})
$$

gegeben sein. Das System aus Gleichung (6) kann aufgeteilt werden in eine Gleichung $\dot{\mathbf{x}}_{c}$, die die nicht-hyperbolischen Eigenwerte enthält und eine Gleichung $\dot{\mathbf{x}}_{h}$, die die hyperbolischen Eigenwerte zusammenfasst

$$
\begin{aligned}
\dot{\mathbf{x}}_{c} & =\mathbf{B} \cdot \mathbf{x}_{c}+f\left(\mathbf{x}_{c}, \mathbf{x}_{h}\right) \\
\dot{\mathbf{x}}_{h} & =\mathbf{C} \cdot \mathbf{x}_{h}+g\left(\mathbf{x}_{c}, \mathbf{x}_{h}\right) .
\end{aligned}
$$

Aus diesem Gleichungssystem folgt der Ausdruck der Zentrumsmannigfaltigkeit

$$
\mathbf{x}_{h}=h\left(\mathbf{x}_{c}\right)
$$

Leitet man die Gleichung (8) mittels Kettenregel nach der Zeit ab ergibt durch Einsetzen in das Gleichungsssystem (7) die zu lösende partielle Differentialgleichung

$$
\frac{\partial h}{\partial \mathbf{x}_{c}} \cdot\left[\mathbf{B} \cdot \mathbf{x}_{c}+f\left(\mathbf{x}_{c}, h\left(\mathbf{x}_{c}\right)\right)\right]=\mathbf{C} \cdot h\left(\mathbf{x}_{c}\right)+g\left(\mathbf{x}_{c}, h\left(\mathbf{x}_{c}\right)\right) .
$$

Diese Gleichung ist für diesen Anwendungsfall nicht explizit lösbar, daher wird der in Gleichung (10) gezeigte Taylorpolynomansatz gewählt, um die Zentrumsmannigfaltigkeit zu bestimmen

$$
h\left(\mathbf{x}_{c}\right)=h\left(x_{1}, x_{2}\right)=a_{20} \cdot x_{1}^{2}+a_{11} \cdot x_{1} x_{2}+a_{02} \cdot x_{2}^{2} .
$$

Das hier gezeigte 5-dimensionale Anwendungsbeispiel liegt nicht in der gewünschten Normalform vor, somit muss das System zunächst mittels linearer Äquivalenztransformation auf die gewünschte Normalform gebracht werden. Aufgrund der Komplexität der semi-symbolischen Transformation ist dieser Schritt nicht trivial und Gegenstand weiterer Forschungsarbeiten. Im nächsten Abschnitt wird das System numerisch gelöst und einige Erkenntnisse der höherdimensionalen Betrachtung präsentiert.

\section{Höherdimensionale Betrachtung}

Die numerische Lösung des 5-dimensionalen Differentialgleichungssystems ist in Abb. 6 gezeigt. Zu erkennen ist das Einlaufen der Lösung im Zustandsraum in einen Grenzzyklus. Zunächst scheint es Überschneidungen der Trajektorie zu geben, was im Zustandsraum nicht möglich ist.

Durch eine Verkippung der Ansicht ist allerdings zu erkennen, dass der Grenzzyklus sich in einer anders orientierten Fläche als der Einschwingvorgang befindet (vgl. Abb. 7). 
$10^{-3}$

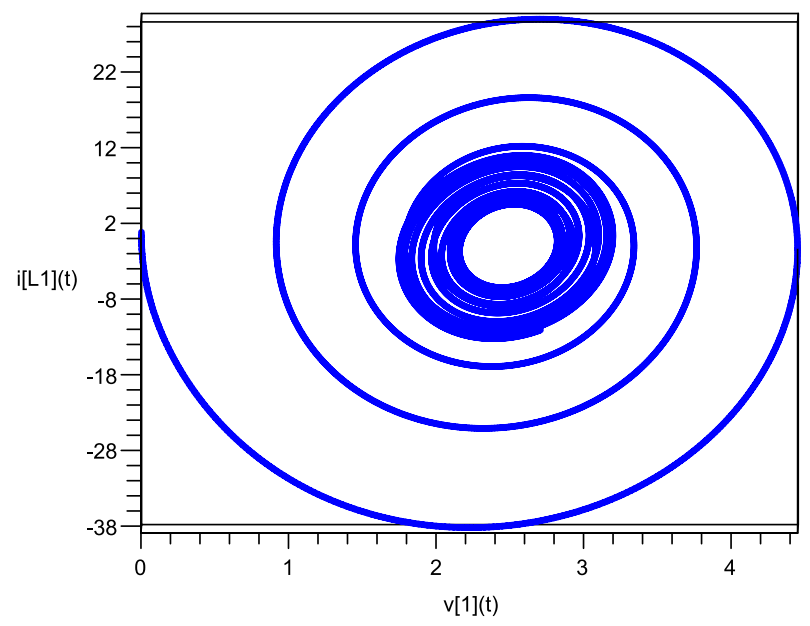

Abb. 6. $v_{C_{p}}$ über $v_{1}$ und $i_{L 1}$, Einlaufen in Grenzzyklus: Ansicht 1 .

Die Betrachtung der Schaltung im höherdimensionalen $\mathrm{Zu}$ standsraum bietet somit die Möglichkeit das Schaltungsverhalten über ausgewählte Zustandsgrößen darzustellen (hier $v_{C p}$ über $v_{1}$ und $i_{L_{1}}$ ) und die Auswirkung asymmetrischer und nichtlinearer Effekte auf das Schaltungsverhalten im $\mathrm{Zu}$ standsraum zu untersuchen.

\section{Zusammenfassung}

In dieser Arbeit wurde ein alternatives und höherdimensionales LC-Tank VCO Modellierungskonzept vorgestellt. Dafür wurde zunächst auf einen gängigen Modellierungsansatz eingegangen und anschließend dessen Stärken und Schwächen erörtert.

$\mathrm{Zu}$ den Stärken des vorgestellten Modellierungskonzeptes gehört vor allem die Möglichkeit die asymmetrischen und nichtlinearen Effekte der Schaltung mit zu berücksichtigen, sowie strukturelle Erweiterungen mit geringem Aufwand in das Differentialgleichungssystem einzubetten. Die Analysen beziehen sich auf eine in Hajimiri (1999) vorgestellten, um eine Filterkapazität erweiterten, NMOS-LC-Tank VCOSchaltung.

Um die Andronov-Hopf-Bifurkationsanalyse anzuwenden, muss das System auf die 2. Ordnung reduziert werden. $\mathrm{Zu}$ diesem Zweck muss das System zunächst in der in Abschnitt 5.1 gezeigten Normalform vorliegen. Im Anschluss dazu wurde die Methode der Zentrumsmannigfaltigkeit als Verfahren zur Ordnungsreduktion vorgestellt. Daraufhin wurden einige Ergebnisse durch numerische Simulation präsentiert.

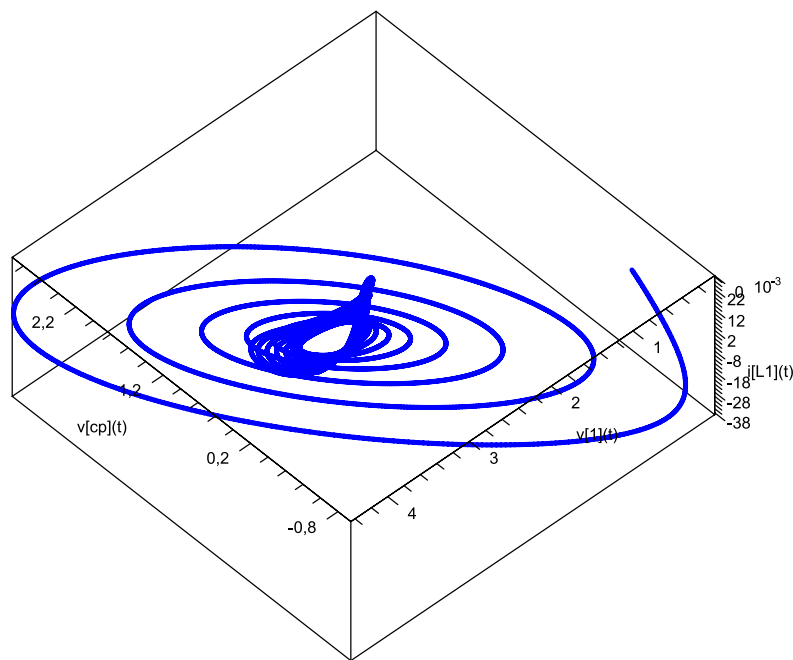

Abb. 7. $v_{C_{p}}$ über $v_{1}$ und $i_{L 1}$, Einlaufen in Grenzzyklus: Ansicht 2.

\section{Literatur}

Bremer, J., Zorn, C., Przytarski, J., and Mathis, W.: A Nonlinear Systematic Design Flow for LC Tank VCOs Based on Large Signal Capacitance Modeling, Proceedings of the IEEE International Symposium on Circuits and Systems ISCAS, Taipei, Taiwan, accepted for publication, 2009.

Buonomo, A. and Lo Schiavo, A.: Modelling and analysis of differential VCOs, Int. J. Circ. Theor. App., 32, 117-131, 2004.

Guckenheimer, J. and Holmes, P.: Nonlinear Oscillations, Dynamical systems and Bifurcations of Vector Fields, Springer Verlag, New York, 123-138, 1983.

Hajimiri, A. and Lee, T. H.: Design Issues in CMOS Differential LC Oszillators, IEEE J. Solid-St. Circ., 34(5), 717-724, Mai 1999.

Hegazi, E. and Abidi, A. A.: Varactor characteristics, oscillator tuning curves, and AM-FM conversion, IEEE J. Solid-St. Circ., 38(6), 896-909, Juni 2003.

Keidies, C. and Mathis, W.: Application of Center Manifolds to Oscillator Analysis, Proc. 12th European Conf. on Circuit Theory and Design (ECCTD'95), Istanbul, Türkei, 1, 447-450, 27-31 August 1995.

Kuznetsov, Y. A.: Elements of Applied Bifurcation Theory, Springer Verlag, New York, 3rd edn., 157-188, 2004.

Mendez, M., Mateo, D., Aragones, X., and Gonzales, J. L.: Phase noise degradation of LC-Tank VCOs due to substrate noise and package coupling, 32st European Solid-State Circuits Conference, 105-108, 12-16 September 2005.

Prochaska, M., Belski, A., and Mathis, W.: Bifurcation Analysis of On-Chip Oscillators, Proceedings of the IEEE International Symposium on Circuits and Systems (ISCAS), Cobe, Japan, 2005.

Soens, C., Van der Plas, G., Wambacq, P., and Donnay, S.: Simulation methodology for analysis of substrate noise impact on ana$\log /$ RF circuits including interconnect resistance, Design, Automation, and Test in Europe, Proceedings of the conference on Design, Automation and Test in Europe, 270-276, 2005.

Yue, C. P. and Wong, S. S.: Physical modeling of spiral inductors on silicon, IEEE T. Electron. Dev., 47(3), 560-568, March 2000. 